ACCepted By ApJ LetTers

Preprint typeset using $\mathrm{LAT}_{\mathrm{EX}}$ style emulateapj v. 14/09/00

\title{
STAR FORMATION RATE FROM DUST INFRARED EMISSION
}

\author{
AKIO K. INOUE \\ Department of Astronomy, Faculty of Science, Kyoto University, Sakyo-ku, Kyoto 606-8502, JAPAN \\ inoue@kusastro.kyoto-u.ac.jp \\ Accepted by ApJ Letters
}

\begin{abstract}
We examine what types of galaxies the conversion formula from dust infrared (IR) luminosity into the star formation rate (SFR) derived by Kennicutt (1998) is applicable to. The ratio of the observed IR luminosity, $L_{\mathrm{IR}}$, to the intrinsic bolometric luminosity of the newly $\left(\lesssim 10 \mathrm{Myr}\right.$ ) formed stars, $L_{\mathrm{SF}}$, of a galaxy can be determined by a mean dust opacity in the interstellar medium and the activity of the current star formation. We find that these parameters area being $0.5 \leq L_{\mathrm{IR}} / L_{\mathrm{SF}} \leq 2.0$ is very large, and many nearby normal and active star-forming galaxies really fall in this area. It results from offsetting two effects of a small dust opacity and a large cirrus contribution of normal galaxies relative to starburst galaxies on the conversion of the stellar emission into the dust IR emission. In conclusion, the SFR determined from the IR luminosity under the assumption of $L_{\mathrm{IR}}=L_{\mathrm{SF}}$ like Kennicutt (1998) is reliable within a factor of 2 for all galaxies except for dust rich but quiescent galaxies and extremely dust poor galaxies.
\end{abstract}

Subject headings: dust, extinction — galaxies: general — infrared: galaxies — stars: formation

\section{INTRODUCTION}

Dust infrared (IR) luminosity ${ }^{1}$ is a familiar indicator of the current star formation rate (SFR). Especially, the most active star-forming galaxies (e.g., Arp 220) emit almost all their radiation energy in the IR range (Soifer, Houck, \& Neugebauer 1987; Sanders \& Mirabel 1996). For such galaxies, Kennicutt (1998a) derived a conversion formula from the IR luminosity to the SFR by assuming the IR luminosity equal to the bolometric luminosity and 10$100 \mathrm{Myr}$ continuous star formation (hereafter K98 IR formula). More recently, Inoue, Hirashita, \& Kamaya (2000; hereafter IHK00) theoretically constructed a new conversion law from IR to SFR, which we can apply not only to dusty starbursts but also to normal (or quiescent) galaxies.

Although the SFRs determined from various indicators should agree with each other, we often find discrepancies among them. Thus, many authors try to resolve such discrepancies (Sullivan et al. 2000; Bell \& Kennicutt 2001; Hopkins et al. 2001; Charlot et al. 2002; Buat et al. 2002; Rosa-González et al. 2002). In this context, the SFR from the IR luminosity via K98 IR formula is assumed to be the correct SFR in spite of the fact that K98 IR formula can be applied to only dusty starbursts in principle (Hopkins et al. 2001; Rosa-González et al. 2002). Indeed, the SFR via K98 IR formula shows a good agreement with that from the $\mathrm{H} \alpha$ luminosity even for samples of normal galaxies if we correct the $\mathrm{H} \alpha$ luminosity for the dust extinction in the interstellar medium (ISM), that in H II regions, and the stellar Balmer absorption (Charlot et al. 2002; RosaGonzález et al. 2002). Also, an empirical conversion factor into the SFR from the IR luminosity for normal late-type spiral galaxies agrees with that of K98 IR formula within a factor of 2 (Buat \& Xu 1996). Why can K98 IR formula present the correct SFR even for normal galaxies?

In this Letter, we examine what types of galaxies K98
IR formula is applicable to, and how much correction factor against the formula is required to obtain a more precise SFR. In $\S 2$, we formulate the relation between the IR luminosity and the star formation (SF) luminosity. In $\S 3$, we describe a model of dust extinction of galaxies. In $\S 4$, we show that the ratio of the IR luminosity to the SF luminosity becomes of order unity within a factor of 2 for a very wide parameter area, and many nearby galaxies really fall in this area.

\section{INFRARED LUMINOSITY OF GALAXIES}

Following IHK00, we formulate the relation between the observed IR luminosity of a galaxy, $L_{\mathrm{IR}}$, and the intrinsic bolometric luminosity of the newly $(\lesssim 10 \mathrm{Myr})$ formed stellar population (hereafter SF population), $L_{\mathrm{SF}}$.

The IR luminosity of a galaxy is expressed by the following:

$$
L_{\mathrm{IR}}=L_{\mathrm{Ly} \alpha}+(1-f) L_{\mathrm{LC}}+\epsilon L_{\mathrm{UV}}+\eta L_{\mathrm{under}}
$$

where $L_{\mathrm{Ly} \alpha}, L_{\mathrm{LC}}, L_{\mathrm{UV}}$, and $L_{\text {under }}$ are luminosities of Lyman $\alpha$ photons generated in $\mathrm{H}$ II regions, Lyman continuum (LC) photons and nonionizing $(\lambda>912 \AA)$ photons emitted by the SF population, and photons from underlying stellar population, respectively. The parameters, $f, \epsilon$, and $\eta$ are a fraction of LC photons contributing to hydrogen ionization, an efficiency of the dust extinction for nonionizing photons from the SF population, and that for photons from underlying population. Here, we assume that all Lyman $\alpha$ photons are eventually absorbed by dust during a number of resonance scatterings in the ISM. We neglect the escape of LC photons from a galaxy, the consumption of LC photons by Helium ionization, any extinctions for hydrogen's recombination lines within $\mathrm{H}$ II regions except for Lyman $\alpha$, and the contribution to the IR luminosity by AGN activity.

${ }^{1}$ In this Letter, the term 'IR' means the whole wavelength range of dust emission. The 'IR luminosity' dose not include the component emitted by stars directly. 
According to the Kurucz ATLAS 9 stellar spectra with Solar metallicity and a turbulence speed of $2 \mathrm{~km} \mathrm{~s}^{-1}$, we obtain $L_{\mathrm{LC}}=0.37 L_{\mathrm{SF}}$ and $L_{\mathrm{UV}}=0.63 L_{\mathrm{SF}}$ if the Salpeter IMF $\left(0.1-100 M_{\odot}\right)$ is assumed. Since about two-thirds of the ionization-recombination processes produce Lyman $\alpha$ photons under case B (Spitzer 1978), we also obtain $L_{\mathrm{Ly} \alpha}=0.12 f L_{\mathrm{SF}}$. Here, we note that only a fraction $f$ of LC photons ionize hydrogen. Moreover, we introduce a new parameter, $\gamma$, to represent the intrinsic stellar luminosity fraction of the SF population to all stellar population; $\gamma=L_{\mathrm{SF}} / L_{\text {total }}$, where $L_{\text {total }}=L_{\mathrm{SF}}+L_{\text {under }}$. This parameter is an indicator of the star-forming activity. Therefore, equation (1) is reduced to

$$
\frac{L_{\mathrm{IR}}}{L_{\mathrm{SF}}}=0.37-0.25 f+0.63 \epsilon+\eta \frac{1-\gamma}{\gamma} .
$$

For dusty starbursts $(\gamma \sim 1)$, the right hand side of equation (2) becomes unity since the dust opacity is enough high to absorb all photons emitted by stars (i.e. $f=0$ and $\epsilon=1)$. In principle, K98 IR formula can be applied to only such galaxies. On the other hand, if we determine all four parameters $(f, \epsilon, \eta$, and $\gamma)$, we can convert the observed IR luminosity of all kinds of galaxies to the SF luminosity, and then, to the current SFR. This is the algorithm of IHK00.

\section{DUST OPACITY OF GALAXIES}

Here, we describe a model of dust extinction to determine the values of $f, \epsilon$, and $\eta$, in equation (2). We will discuss in $\S 4.2$ the effect of the choice of a specific extinction law on our result.

Following Charlot \& Fall (2000) (especially their Fig. 1 ), we consider, first, two types of dust extinction. One is the extinction by diffuse ISM dust $\left(\tau_{\lambda}^{\mathrm{ISM}}\right)$, and the other is by dust accompanying the birth clouds of young stars $\left(\tau_{\lambda}^{\mathrm{BC}}\right)$. Then, we assume $\tau_{\lambda}^{\mathrm{ISM}}=\tau_{V}^{\mathrm{ISM}}\left(\lambda / \lambda_{V}\right)^{-n}$ and $\tau_{\lambda}^{\mathrm{BC}}=\mu \tau_{V}^{\mathrm{ISM}}\left(\lambda / \lambda_{V}\right)^{-n}$ for $\lambda>912 \AA$, where $\mu=2$, $n=0.7$, and $\lambda_{V}=5500 \AA$ are adopted, so as to reproduce the observed relation between UV flux slope and luminosity ratio of IR to UV for nearby star-forming galaxies (Charlot \& Fall 2000). We define the dust optical depth for the SF population as $\tau_{\lambda}^{\mathrm{SF}}=\tau_{\lambda}^{\mathrm{BC}}+\tau_{\lambda}^{\mathrm{ISM}}$.

For simplicity, we assume that the dust geometry is an uniform screen (or the optical depth defined above is an effective one). Then, we define $\epsilon$ as an average of $\epsilon(t)$ over the age range of $0-10 \mathrm{Myr}$, where $\epsilon(t)=$ $1-\int L_{\lambda}(t) \exp \left(-\tau_{\lambda}^{\mathrm{SF}}\right) d \lambda / \int L_{\lambda}(t) d \lambda$ and $L_{\lambda}(t)$ is the luminosity density of a simple stellar population (SSP) with an age $t$. We use SSPs produced by PÉGASE 2.0 (Fioc \& Rocca-Volmerange 1997) throughout this Letter. In the same way, we define $\eta$ as an average of $\eta(t)$ over the age of $10 \mathrm{Myr}-15$ Gyr but $\tau_{\lambda}^{\mathrm{SF}}$ is replaced with $\tau_{\lambda}^{\mathrm{ISM}}$. The obtained $\epsilon$ and $\eta$ are a function of only $\tau_{V}^{\mathrm{ISM}}$, the normalization of the adopted extinction law.

Let us introduce one more type of dust extinction to determine the parameter $f$, Lyman continuum extinction (Inoue, Hirashita, \& Kamaya 2001, Inoue 2001), which is the extinction for LC photons by dust in $\mathrm{H}$ II regions before LC photons ionize neutral hydrogens. The parameter $f$ is determined from the dust optical depth for LC photons in $\mathrm{H}$ II regions, $\tau_{L C}^{\mathrm{HII}}$, via eq. (8) in Petrosian, Silk, \& Field (1972). Here, we assume a scaling law between $\tau_{L C}^{\text {HII }}$ and $\tau_{V}^{\mathrm{ISM}}$, i.e., $\tau_{L C}^{\mathrm{HII}} / \tau_{V}^{\mathrm{ISM}} \equiv \xi$.

Now, we attempt to estimate $\xi$ from real data. We have shown that $\tau_{L C}^{\mathrm{HII}}$ is proportional to the dust-to-gas mass ratio, $\mathcal{D}$, in Inoue et al. (2001). Here, we adopt the relation, $\tau_{L C}^{\mathrm{HII}} \simeq\left(\mathcal{D} / \mathcal{D}_{\mathrm{MW}}\right)($ Inoue 2001$)$, where $\mathcal{D}_{\mathrm{MW}}=6 \times 10^{-3}$ is a typical Galactic value (Spitzer 1978). We estimated $\mathcal{D}$ for seven nearby spiral galaxies observed by ISO (Alton et al. 1998) in Inoue et al. (2001). The mean value is $1.8 \times 10^{-3} .^{2}$ On the other hand, we can estimate the dust optical depth from the IR surface brightness. By neglecting the self-absorption of the IR photons and assuming the obtained optical depth to correspond to that in the diffuse ISM, we obtain $\tau_{V}^{\mathrm{ISM}}=0.36$ as a mean dust optical depth for these seven galaxies, where we adopt the dust emissivity determined by Bianchi, Davies, \& Alton (1999). Therefore, we obtain $\xi \approx 1$.

Although the real relation between $\tau_{L C}^{\mathrm{HII}}$ and $\tau_{V}^{\mathrm{ISM}}$ is quite uncertain, we find in equation (2) that the effect of $f$ on the ratio $L_{\mathrm{IR}} / L_{\mathrm{SF}}$ is small. Indeed, the effect of the deviation from $f=0.5$ on the $L_{\mathrm{IR}} / L_{\mathrm{SF}}$ is at most \pm 0.125 .

\section{LUMINOSITY RATIO OF DUST INFRARED EMISSION TO STAR-FORMING POPULATION}

Now, we can determine $L_{\mathrm{IR}} / L_{\mathrm{SF}}$ from only two parameters; a mean dust opacity $\left(\tau_{V}^{\mathrm{ISM}}\right)$ and a star-forming activity $(\gamma)$. We survey these parameters space to clarify when we can determine the SFR from $L_{\mathrm{IR}}$ within a factor of 2 uncertainty via K98 IR formula, i.e., when $0.5 \leq L_{\mathrm{IR}} / L_{\mathrm{SF}} \leq 2.0$ is satisfied.

In Figure 1, we show some constant $L_{\mathrm{IR}} / L_{\mathrm{SF}}$ lines in the $\tau_{V}^{\mathrm{ISM}}-\gamma$ plane. We define the 'applicable area' as the parameter area satisfying the condition $0.5 \leq L_{\mathrm{IR}} / L_{\mathrm{SF}} \leq$ 2.0. The 'applicable area' is between two thick lines in Figure 1 since the upper thick line is the case of $L_{\mathrm{IR}} / L_{\mathrm{SF}}=0.5$ and the lower one is the case of $L_{\mathrm{IR}} / L_{\mathrm{SF}}=2.0$. Also, the thin line in Figure 1 represents the case of $L_{\mathrm{IR}} / L_{\mathrm{SF}}=1.0$. We find that the 'applicable area' is very wide in the $\tau_{V}^{\mathrm{ISM}}$ $\gamma$ plane. This is a main conclusion in this Letter.

For a galaxy with an exponentially declining star formation history (SFH) of $\gtrsim 10$ Gyr time scale, age of $\gtrsim 1$ Gyr, and Salpeter IMF, we find that $\gamma \sim 0.5$. Thus, many spiral and irregular galaxies are likely to fall in the 'applicable area'. In order to examine whether real galaxies fall in the 'applicable area' or not, we will estimate their $\gamma$ and $\tau_{V}^{\mathrm{ISM}}$ in the following. Then, we will discuss implications from our results.

\subsection{Estimation of $\gamma$ and $\tau_{V}^{\mathrm{ISM}}$}

First, we estimate $\gamma$ of galaxies from the observed equivalent width of $\mathrm{H} \alpha$ emission line corrected for dust and stellar absorption effects. By the definition of $\gamma$, we can express $\gamma=E W_{\mathrm{H} \alpha}\left(C_{\mathrm{H} \alpha} / C_{R}\right)$, where $E W_{\mathrm{H} \alpha}$ is the intrinsic equivalent width of $\mathrm{H} \alpha$ emission line, $C_{\mathrm{H} \alpha}$ is the intrinsic ratio of the bolometric luminosity to the $\mathrm{H} \alpha$ emission line luminosity of the SF population, and $C_{R}$ is the intrinsic ratio of the bolometric luminosity to the luminosity density

2 The mean value is different from that in Inoue et al. (2001) because we correct it for He contribution and for the calibration error of the flux density at $200 \mu \mathrm{m} \mathrm{(-30 \% ;} \mathrm{Alton} \mathrm{et} \mathrm{al.} \mathrm{1998).} \mathrm{In} \mathrm{addition,} \mathrm{we} \mathrm{should} \mathrm{note} \mathrm{that} \mathcal{D}_{\mathrm{MW}}$ by Spitzer (1978) is estimated from the analysis of the extinction properties for a number of line of sights, which is different from our method. 
at $R$-band of all stellar population.

If we specify the metallicity and IMF of galaxies, the value of $C_{\mathrm{H} \alpha}$ is determined from only the electron temperature and density in $\mathrm{H}$ II regions under case $\mathrm{B}$ approximation. On the contrary, the value of $C_{R}$ depends on the SFH and age of galaxies as well as the metallicity and IMF. We examine the evolution of $C_{R}$ with the galactic age, so that we find that the factor $C_{\mathrm{H} \alpha} / C_{R}$ falls in the range of $0.002-0.004$ unless we consider a galaxy younger than a few Gyr or a galaxy with a short ( $\lesssim 1$ Gyr) exponential time scale. Thus, we adopt a constant 0.003 as $C_{\mathrm{H} \alpha} / C_{R}$, which corresponds to the case of Solar metallicity, exponentially declining SFH with 10 Gyr time scale, and about 13 Gyr age. Here, we do not take into account the chemical enrichment effect.

Therefore, we estimate $\gamma$ from the following equation approximately;

$$
\gamma=\left\{\begin{array}{ll}
0.003 E W_{\mathrm{H} \alpha} & \text { for } E W_{\mathrm{H} \alpha} \leq 333 \AA \\
1 & \text { for } E W_{\mathrm{H} \alpha}>333 \AA
\end{array},\right.
$$

where the second case is needed due to $\gamma \leq 1$ by its definition. In the real case, the value of $\gamma$ asymptotically approaches unity by decreasing the factor $C_{\mathrm{H} \alpha} / C_{R}$, when $E W_{\mathrm{H} \alpha}$ increases. Thus, we overestimate $\gamma$ for a galaxy with a very large $E W_{\mathrm{H} \alpha}$. However, the real value of $\gamma$ is about unity for such galaxies. On the other hand, we underestimate $\gamma$ for a galaxy with a very small $E W_{\mathrm{H} \alpha}$. We confirm that this effect is also not so large (at most factor of 2).

Although the effect of the change of the metallicity on $C_{\mathrm{H} \alpha} / C_{R}$ is small, that of the IMF slope is large. If we choose the IMF slope of 1.35 (Salpeter's slope is 2.35 ), a typical $C_{\mathrm{H} \alpha} / C_{R}$ decreases an order of magnitude, about 0.0003 . In the case of the slope of 3.35 , we find $C_{\mathrm{H} \alpha} / C_{R} \simeq 0.007$ typically. This is because a top-heavy IMF (1.35 slope) results in a smaller fraction of the $R$ band luminosity density in the bolometric luminosity since massive stars do not contribute to the $R$-band light so effectively. A steep IMF provides an opposite result.

Now, we must estimate the intrinsic equivalent width, $E W_{\mathrm{H} \alpha}$, from the observed one, $E W_{\mathrm{H} \alpha}^{\mathrm{obs}}$. Assuming that the underlying stellar population (age $\gtrsim 10 \mathrm{Myr}$ ) dominates the $R$-band continuum and the stellar Balmer absorption, we can express

$$
E W_{\mathrm{H} \alpha}=\left(E W_{\mathrm{H} \alpha}^{\mathrm{obs}}+E W_{\mathrm{H} \alpha}^{*, \mathrm{abs}}\right) \frac{1}{f} \exp \left(\tau_{\lambda_{\mathrm{H} \alpha}}^{\mathrm{SF}}-\tau_{\lambda_{\mathrm{H} \alpha}}^{\mathrm{ISM}}\right),
$$

where $E W_{\mathrm{H} \alpha}^{*, a b s}$ is the equivalent width of the stellar absorption, and $\lambda_{\mathrm{H} \alpha}=6563 \AA$. The factor $1 / f$ means that only a fraction $f$ of LC photons is used to ionize neutral hydrogen atoms.

Next, we estimate $\tau_{V}^{\mathrm{SF}}$ from the Balmer decrement corrected for the stellar absorption by assuming $E W_{\mathrm{H} \alpha}^{* \text { abs }}=$ $E W_{\mathrm{H} \beta}^{*, \text { abs }}=5 \AA$, which noted by Kennicutt (1992) as a typical value, and then, we convert the obtained $\tau_{V}^{\mathrm{SF}}$ into $\tau_{V}^{\text {ISM }}$ by using the parameter $\mu=2$ (See $\S 3$ ). Here, we also assume the electron temperature of $10^{4} \mathrm{~K}$ and case B. Once the optical depth $\tau_{V}^{\text {ISM }}$ is obtained, we can estimate $f$ parameter, $E W_{\mathrm{H} \alpha}$ from $E W_{\mathrm{H} \alpha}^{\mathrm{obs}}$, and then, $\gamma$. The obtained values of $\tau_{V}^{\text {ISM }}$ are systematically smaller than but roughly consistent with those determined from the UV slope method (Meurer, Heckman, \& Calzetti 1999) for sample galaxies described below.
The uncertainty of $\tau_{V}^{\mathrm{ISM}}$ from the Balmer decrement is large, $\sim \pm 0.2$. As a result, the uncertainty of the obtained $\gamma$ is $\sim \pm 0.2$, which is estimated from a simple Monte Carlo simulation if we do not take account of the uncertainties of the adopted parameters of the dust extinction law (i.e., $\eta, \xi$, and $n$ in $\S 3$ ). Also, we find that a random error of $\tau_{V}^{\text {ISM }}$ causes a somewhat overestimation of $\gamma$. To display this point, we show a typical error bar in Figure 1. In addition, we select only galaxies with enough strong $\mathrm{H}$ $\beta$ 'emission' line to obtain a reliable Balmer decrement. Thus, our sample galaxies are biased towards larger $\gamma$.

One might think that the parameter $\gamma$ can be estimated from the ratio of the UV luminosity and the $R$ or $K$-band luminosity. However, we can do it reasonably only if we specify the SFH and age of galaxies. That is, the coefficient between $\gamma$ and $L_{U V} / L_{R}$ or $L_{U V} / L_{K}$ varies together with the galactic age significantly. This is because a significant fraction of the UV light originates in the underlying stellar population with its age of $\gtrsim 10 \mathrm{Myr}$. On the contrary, the ionizing photons producing the $\mathrm{H} \alpha$ photons originate in almost only the SF population (its age $\lesssim 10 \mathrm{Myr}$ ). Therefore, we estimated $\gamma$ from the equivalent width of the $\mathrm{H} \alpha$ line.

\subsection{Results \& Discussions}

To plot real galaxies in Figure 1, we compile all morphological types of galaxies except for elliptical galaxies from the literature. In Figure 1, the filled circles are early type spiral (Sa-Sab) galaxies observed by Usui, Saitō, \& Tomita (2001), who selected active star-forming early type spiral galaxies with $\log \left(L_{\mathrm{FIR}} / L_{B}\right) \geq 0.5$. The filled squares, open squares, and crosses are early type spiral (S0-Sbc), late type spiral $(\mathrm{Sc}-\mathrm{Sm})$, and irregular (including peculiar and merger) galaxies, respectively, observed by Kennicutt (1992), who selected galaxies covering all morphological types. The filled triangles, open triangles, and stars are also early spiral, late spiral, and irregular (also peculiar and merger) galaxies, respectively, observed by StorchiBergmann, Kinney, \& Challis (1995), who selected active star-forming galaxies observed by $I U E$ satellite. Any galaxies containing an AGN are excluded.

We find that almost all $(43 / 52)$ galaxies fall in the 'applicable area'. For 7 out of 9 galaxies in the outside of the 'applicable area', we fail to determine $\tau_{V}^{\mathrm{ISM}}$ because they become negative values. Although the uncertainties of the determined $\tau_{V}^{\text {ISM }}$ and $\gamma$ are large and compiled galaxies do not make a complete sample, this result indicate that many nearby normal galaxies are really in the 'applicable area'.

We emphasize the following two points. First, it is a just coincidence that K98 IR formula is applicable to normal galaxies. It results from offsetting two effects of a small dust opacity and a large cirrus contribution of normal galaxies relative to starburst galaxies on the conversion coefficient of K98 IR formula (Kennicutt 1998b, IHK00). For an example case of a galaxy with $\tau_{V}^{\mathrm{ISM}}=0.3$ and $E W_{\mathrm{H} \alpha}^{\mathrm{obs}}=50 \AA$, while only about $70 \%$ luminosity of the $\mathrm{SF}$ population is converted into the IR luminosity, it is almost the same amount as the rest $30 \%$ SF luminosity that is supplied with the IR luminosity by the underlying population. 
Second, the result above is robust for changing the adopted parameter sets of the dust extinction. One might think that our result depends on the dust extinction law adopted. However, we note that it dose not change when we change the parameters in the range of $0 \leq \mu \leq 3$, $0.4 \leq n \leq 1.0$, and $0 \leq \xi \leq 2$ as shown in Figure 2 (an extra figure). This point will be discussed in detail in the forthcoming paper (Inoue 2002, in preparation). ${ }^{3}$

We discuss the condition required to fall in the 'applicable area'. From the lower bound of $\gamma$ in Figure 1 $\left(L_{\mathrm{IR}} / L_{\mathrm{SF}}=2.0\right)$, we find that a quiescent galaxy with a large dust amount (a small $\gamma$ and a large $\tau_{V}^{\text {ISM }}$ ) is in the outside of the 'applicable area'. Since galaxies showing a lower activity of the star formation tend to be more transparent (Wang \& Heckman 1996; Hopkins et al. 2001), the number of galaxies falling in the area of large $\tau_{V}^{\text {ISM }}$ and small $\gamma$ may be small. Moreover, we find that the lower bound of $\gamma$ is $\sim 0.1$ for a galaxy with $\tau_{V}^{\text {ISM }} \sim 0.3$. This corresponds to $E W_{\mathrm{H} \alpha}^{\mathrm{obs}} \gtrsim 10 \AA$. This boundary can be exceeded not only by active star-forming galaxies but also by many nearby normal spiral and irregular galaxies (Kennicutt 1983).

The upper bound of $\gamma$ in Figure $1\left(L_{\mathrm{IR}} / L_{\mathrm{SF}}=0.5\right)$ reaches unity if $\tau_{V}^{\mathrm{ISM}}$ is larger than about 0.1 . Thus, a starburst galaxy having very small dust content of $\tau_{V}^{\text {ISM }} \lesssim 0.1$ is in the outside of the 'applicable area'. For example, I Zw 18 , which is an active star-forming and the most metal deficient nearby dwarf galaxies, has typically $A_{V}=0.2 \mathrm{mag}$ in the southeast $\mathrm{H}$ II region (Cannon et al. 2002). It corresponds to $\tau_{V}^{\mathrm{ISM}} \sim 0.06$ if we adopt $\mu=2$. By taking into account $\gamma \sim 1$ estimated from very large $E W_{\mathrm{H} \alpha}^{\mathrm{obs}} \sim 1000$ $\AA$ (Cannon et al. 2002), I Zw 18 is in the outside of the 'applicable area'.

In conclusion, we find that the range of a dust opacity and a star-forming activity to be $0.5 \leq L_{\mathrm{IR}} / L_{\mathrm{SF}} \leq 2.0$ is very large. Furthermore, we confirm that these parameters of many nearby normal and active star-forming galaxies really fall in this range. Therefore, we can apply K98 IR formula assuming $L_{\mathrm{IR}}=L_{\mathrm{SF}}$ to many nearby normal galaxies as well as dusty starbursts without any correction factor within a factor of 2 uncertainty. However, we cannot apply it to dust rich but quiescent galaxies and extremely dust poor galaxies. Therefore, we need to divide the coefficient of K98 IR formula by a correction factor, $L_{\mathrm{IR}} / L_{\mathrm{SF}}$, when we apply it to such galaxies. Following the model in this Letter, we can estimate the correction factor for any galaxies if we have all data required, although we must more refine the model.

The author would like to thank H. Kamaya, T. T. Takeuchi, and H. Hirashita for their suggestions to improve the quality of this work, and also, to thank an anonymous referee for his/her many helpful comments to this paper. The author has made extensive use of NASA's Astrophysics Data System Abstract Service (ADS).

\section{REFERENCES}

Alton, P. B., Trewhella, M., Davies, J. J., Evans, R., Bianchi, S., Gear, W., Thronson, H., Valentijn, E., \& Witt, A. 1998, A\&A, 335,807

Bell, E., \& Kennicutt, R. C. 2001, ApJ, 548, 681

Bianchi, S., Davies, J. I., \& Alton, P. B.

Buat, V., \& Xu, C. 1996, A\&A, 306, 61

Buat, V., Boselli, A., Gavazzi, G., \& Bonfanti, C. 2002, A\&A, 383, 801

Cannon, J. M., Skillman, E. D., Garnett, D. R., \& Dufour, R. J. 2002, ApJ, 565, 931

Charlot, S., \& Fall, S. M. 2000, ApJ, 539, 718

Charlot, S., Kauffmann, G., Longhetti, M., Tresse, L., White, S. D. M., Maddox, S. J., \& Fall, S. M. 2002, MNRAS, 330, 876

Fioc, M., \& Rocca-Volmerange, B. 1997, A\&A, 326, 950

Hopkins, A. M., Connolly, A. J., Haarsma, D. B., \& Cram, L. E. 2001, AJ, 122, 288

Inoue, A. K., Hirashita, H., \& Kamaya, H. 2000, PASJ, 52, 539 (IHK00)

Inoue, A. K., Hirashita, H., \& Kamaya, H. 2001, ApJ, 555, 613

Inoue, A. K. 2001, AJ, 122, 1788
Kennicutt, R. C. 1983, ApJ, 272, 54

Kennicutt, R. C. 1992, ApJ, 388, 310

Kennicutt, R. C. 1998a, ApJ, 498, 541 (K98)

Kennicutt, R. C. 1998b, ARA\&A, 36, 189

Meurer, G. R., Heckman, T. M. \& Calzetti, D. 1999, ApJ, 521, 64

Petrosian, V., Silk, J., \& Field, G. B. 1972, ApJ, 177, L69

Rosa-González, D., Terlevich, E., \& Terlevich, R. 2002, MNRAS, in press (astro-ph/0112556)

Sanders, D. B., \& Mirabel, I. F. 1996, ARA\&A, 34, 749

Soifer, B. T., Houck, J. R., \& Neugebauer, G. 1987, ARA\&A, 25, 187

Spitzer, L. 1978, Physical Processes in the Interstellar Medium (New York: Wiley)

Storchi-Bergmann, T., Kinney, A. L., \& Challis, P. 1995, ApJS, 98, 103

Sullivan, M., Treyer, M. A., Ellis, R. S., Bridges, T. J., Milliard, B., \& Donas, J. 2000, MNRAS, 312, 442

Usui, T., Saitō, M., \& Tomita, A. 2001, AJ, 121, 2483

Wang, B., \& Heckman, T. M. 1996, ApJ, 457, 645 


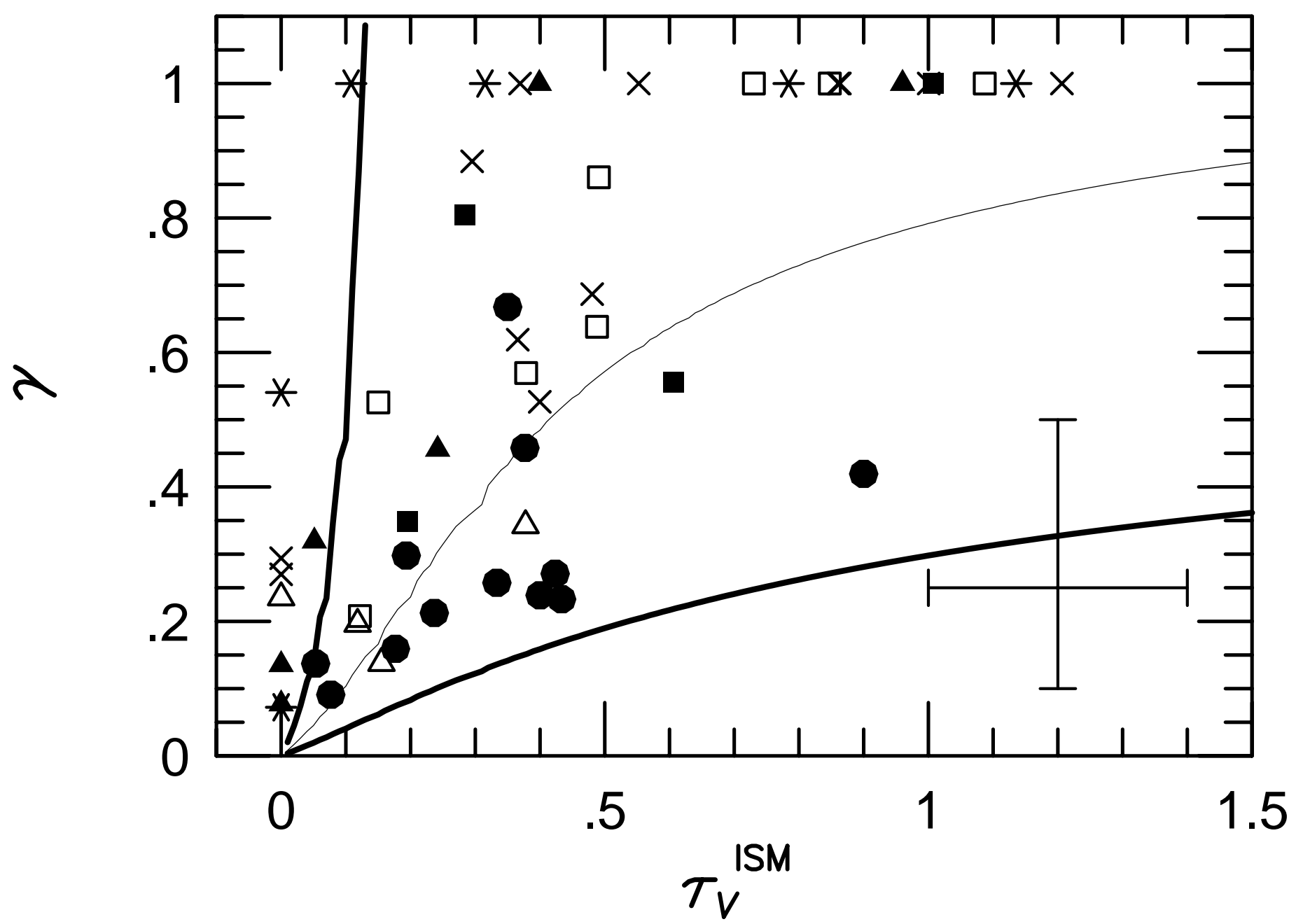

FIG. 1.- Iso- $L_{\mathrm{IR}} / L_{\mathrm{SF}}$ lines in the $\tau_{V}^{\mathrm{ISM}}-\gamma$ plane, where $L_{\mathrm{IR}}$ is the observed total IR luminosity, $L_{\mathrm{SF}}$ is the intrinsic bolometric luminosity of newly $\left(\leqslant 10 \mathrm{Myr}\right.$ ) formed stellar population, $\tau_{V}^{\text {ISM }}$ is a mean optical depth by dust in the diffuse interstellar medium, and $\gamma$ is the luminosity fraction of newly formed stellar population. The upper thick line is $L_{\mathrm{IR}} / L_{\mathrm{SF}}=0.5$, and the lower thick line is $L_{\mathrm{IR}} / L_{\mathrm{SF}}=2.0$. The thin line is $L_{\mathrm{IR}} / L_{\mathrm{SF}}=1.0$. The filled circles are early type spiral (Sa-Sab) galaxies taken from Usui, Saitō, \& Tomita (2001). The filled squares, open squares, and crosses are early type spiral $(\mathrm{S} 0-\mathrm{Sbc}$ ), late type spiral $(\mathrm{Sc}-\mathrm{Sm})$, and irregular (including peculiar and merger) galaxies, respectively, taken from Kennicutt (1992). The filled triangles, open triangles, and stars are also early, late spirals, and irregular galaxies, respectively, taken from Storchi-Bergmann, Kinney, \& Challis (1995). The classifications are taken from NASA/IPAC Extragalactic Database. No galaxies with an AGN are included. A typical error bar is shown in the bottom right of the panel. 

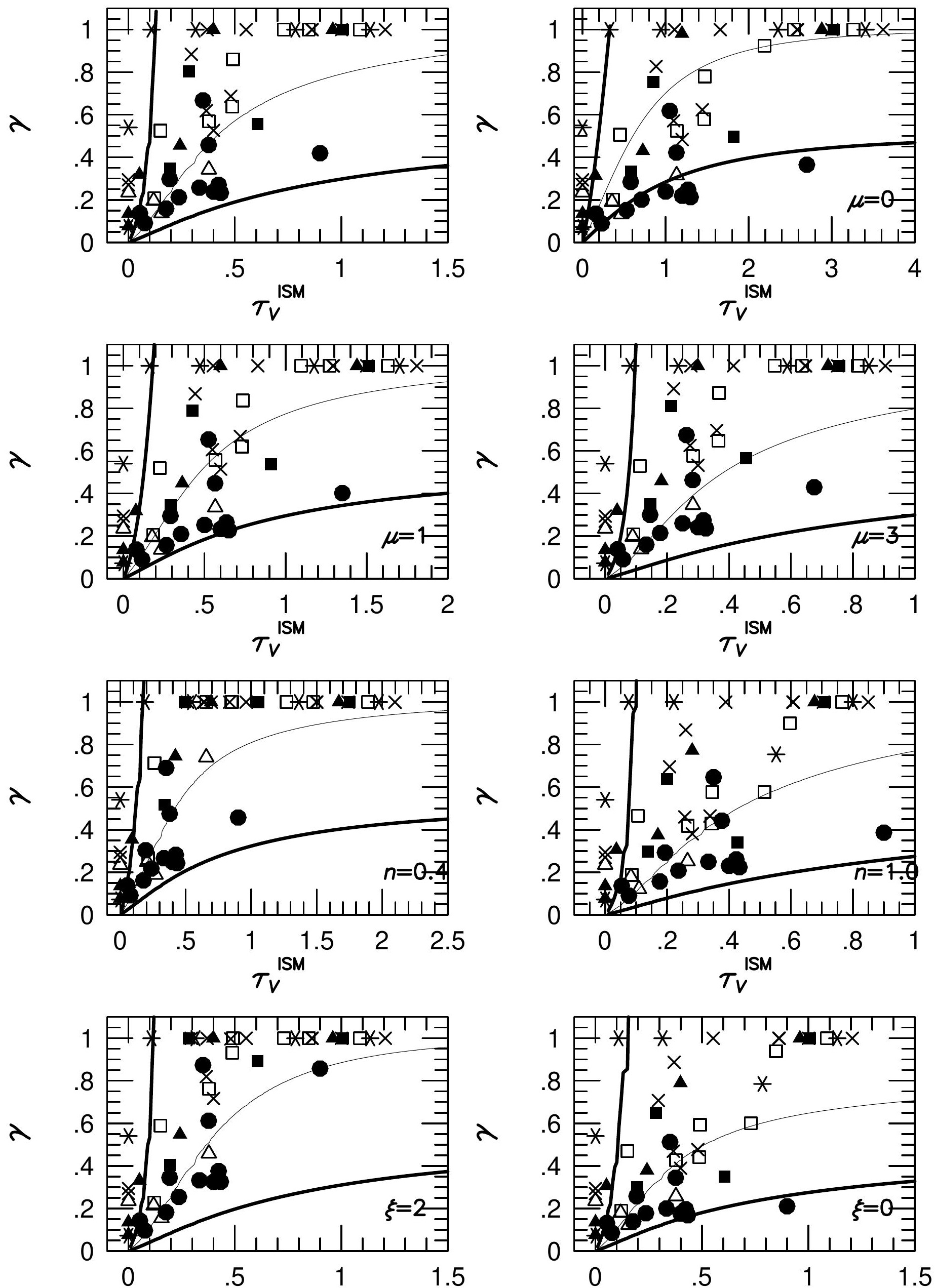